\title{
Direct evidence of a full dipole flip during the magnetic cycle of a sun-like star
}

\author{
S. Boro Saikia ${ }^{1}$, T. Lueftinger ${ }^{1}$, S. V. Jeffers ${ }^{2}$, C. P. Folsom ${ }^{3,4}$, V. See , P. Petit $^{3,4}$, S. C. Marsden ${ }^{6}$, A. A. Vidotto ${ }^{7}$,
} J. Morin ${ }^{8}$, A. Reiners ${ }^{2}$, M. Guedel ${ }^{1}$, and the BCool Collaboration

1 University of Vienna, Department of Astrophysics, Türkenschanzstrasse 17, 1180 Vienna, Austria e-mail: sudeshna.boro.saikia@univie.ac.at

2 University of Goettingen, Institute for Astrophysics, Friedrich Hund Platz 1, 37077 Goettingen, Germany

${ }^{3}$ Université de Toulouse, UPS-OMP, IRAP, Toulouse, France

${ }^{4}$ CNRS, Institut de Recherche en Astrophysique et Planetologie, 14, avenue Edouard Belin, 31400 Toulouse, France

5 University of Exeter, Department of Physics \& Astronomy, Stocker Road, Devon, Exeter EX4 4QL, UK

${ }^{6}$ University of Southern Queensland, Centre for Astrophysics, Toowoomba, QLD 4350, Australia

7 School of Physics, Trinity College Dublin, University of Dublin, Dublin-2, Ireland

${ }^{8}$ LUPM-UMR 5299, CNRS \& Université Montpellier, place Eugène Bataillon, 34095 Montpellier Cedex 05, France

Received 30 September 2018 / Accepted 22 November 2018

\begin{abstract}
Context. The behaviour of the large-scale dipolar field, during a star's magnetic cycle, can provide valuable insight into the stellar dynamo and associated magnetic field manifestations such as stellar winds.

Aims. We investigate the temporal evolution of the dipolar field of the $\mathrm{K}$ dwarf $61 \mathrm{Cyg}$ A using spectropolarimetric observations covering nearly one magnetic cycle equivalent to two chromospheric activity cycles.

Methods. The large-scale magnetic field geometry is reconstructed using Zeeman Doppler imaging, a tomographic inversion technique. Additionally, the chromospheric activity is also monitored.

Results. The observations provide an unprecedented sampling of the large-scale field over a single magnetic cycle of a star other than the Sun. Our results show that 61 Cyg A has a dominant dipolar geometry except at chromospheric activity maximum. The dipole axis migrates from the southern to the northern hemisphere during the magnetic cycle. It is located at higher latitudes at chromospheric activity cycle minimum and at middle latitudes during cycle maximum. The dipole is strongest at activity cycle minimum and much weaker at activity cycle maximum.

Conclusions. The behaviour of the large-scale dipolar field during the magnetic cycle resembles the solar magnetic cycle. Our results are further confirmation that $61 \mathrm{Cyg} \mathrm{A}$ indeed has a large-scale magnetic geometry that is comparable to the Sun's, despite being a slightly older and cooler K dwarf.
\end{abstract}

Key words. stars: magnetic field - stars: solar-type - stars: imaging

\section{Introduction}

In recent years, with the advances made in stellar magnetic field observations, the technique of Zeeman Doppler imaging (ZDI; Semel 1989; Brown et al. 1991; Donati \& Brown 1997; Piskunov \& Kochukhov 2002; Kochukhov \& Piskunov 2002) has been able to reveal the diversity in stellar largescale magnetic field geometry. Both single-epoch studies (Petit et al., in prep.) and long-term monitoring (Fares et al. 2009, 2017; Morgenthaler et al. 2012; Jeffers et al. 2014, 2017; Boro Saikia et al. 2015) have revealed that stellar magnetic geometries do not necessarily have the same large-scale structure as the Sun. While older slowly rotating stars exhibit a more sun-like, almost purely poloidal large-scale magnetic field (Petit et al. 2008), younger rapidly rotating stars can have magnetic geometries that do not bear resemblance to the solar largescale field (Morgenthaler et al. 2012; Boro Saikia et al. 2015; Rosén et al. 2016; Hackman et al. 2016). Surprisingly, until now, sun-like magnetic geometry evolution has been an anomaly, not the expected norm ${ }^{1}$. To date, approximately 20 stars have been observed over multi-epochs, the majority of which were observed by the BCool Collaboration ${ }^{2}$ (Marsden et al. 2014). Of these stars only a few exhibit polarity flips (Petit et al. 2009; Morgenthaler et al. 2011; Fares et al. 2009; Mengel et al. 2016; Boro Saikia et al. 2016; Jeffers et al. 2018), and only 61 Cyg A (Boro Saikia et al. 2016) and $\tau$ Boo (Jeffers et al. 2018) are known to exhibit polarity flips of the large-scale field in phase with the star's chromospheric activity cycle. However, no star has been observed over a full magnetic cycle (or two consecutive activity cycles); one solar magnetic cycle is the time taken by the polarity of the large-scale field to switch to an opposite polarity and flip back to the original

\footnotetext{
1 A solar-type star has a sun-like field evolution when the large-scale magnetic field flips polarity in sync with its activity cycle. Additionally, the large-scale field changes field complexity during the activity cycle in the same way as the Sun does.

2 https://bcool.irap.omp.eu/
} 
sign. This work, for the first time, investigates the evolution of the large-scale field of a sun-like star over its magnetic cycle.

The K dwarf 61 Cyg A was observed for a full activity cycle or half a magnetic cycle in Paper I (Boro Saikia et al. 2016). ZDI reconstructions of this star have revealed a magnetic field geometry that flips its polarity in sync with its chromospheric activity cycle. The field geometry is dominantly poloidal. During activity minimum the poloidal field is strongly dipolar, and at activity maximum quadrupolar and octupolar fields dominate. In the Sun, the dipolar field is known to undergo spatial and temporal evolution during the solar magnetic cycle (Sanderson et al. 2003; DeRosa et al. 2012; Vidotto et al. 2018). The evolution of the 61 Cyg A large-scale field is similar to the evolution of the solar large-scale field over its activity cycle. However, the star was observed for only one activity cycle in Paper I and it could not be established whether such evolution is repeated periodically. If $61 \mathrm{Cyg} \mathrm{A}$ has a sun-like dynamo process operating like that of the present-day Sun, then the strength and location of the dipolar field should have a sun-like evolution during the full magnetic cycle. In this work, the large-scale field of 61 Cyg A is monitored, with strong emphasis on the dipole field, for almost a full magnetic cycle by combining new multi-epoch observations (activity cycle II) with epochs from Paper I (activity cycle I).

\section{Observations}

Simultaneous Stokes $V$ (circularly polarised) and Stokes $I$ (unpolarised) spectra were taken using the high-resolution spectropolarimeter NARVAL at Telescope Bernard Lyot (TBL), Pic du Midi (Aurière 2003). Six epochs of observations were obtained spanning three years (2015.77, 2015.91, 2016.50, 2017.50, 2017.89, 2018.52). Epochs 2017.50, 2017.89, and 2018.52 were observed as part of the BCool Collaboration. We also use data published in Paper I, where six epochs were observed over nine years. Furthermore, epoch 2005.47 was observed as part of POLARBASE (Petit et al. 2014). However, care should be taken when interpreting the 2005.47 results as the rotational phase coverage is sparse.

To detect Zeeman signatures in Stokes $V$, the multi-line technique least-squares deconvolution (LSD; Donati et al. 1997; Kochukhov et al. 2010) is applied, which boosts the signal-tonoise ratio. The LSD Stokes $V$ and Stokes $I$ profiles are obtained following the same procedure as applied in Paper I.

\section{Methods}

Zeeman Doppler imaging is the only technique that can reconstruct the large-scale surface magnetic geometry in sun-like stars. It is a tomographic technique that inverts LSD Stokes $V$ profiles to reconstruct the magnetic maps. The code iteratively fits synthetic Stokes profiles obtained from a stellar model to observed Stokes spectra, until the desired reduced $\chi^{2}$ is reached (see Donati et al. 2006; Folsom et al. 2016, and the references therein for more details on the ZDI technique). Since it is an inverse problem, the maximum entropy technique is utilised as a regularisation tool (Skilling \& Bryan 1984).

The stellar parameters (inclination $=70^{\circ}, \quad v \sin i=$ $0.92 \mathrm{~km} \mathrm{~s}^{-1}$ ) used to reconstruct the magnetic maps are taken from Paper I. A rotation period of 34.2 days and HJD 2454308.49809 as zero phase are used to calculate the phases (taken from Paper I). The maximum spherical harmonic degree

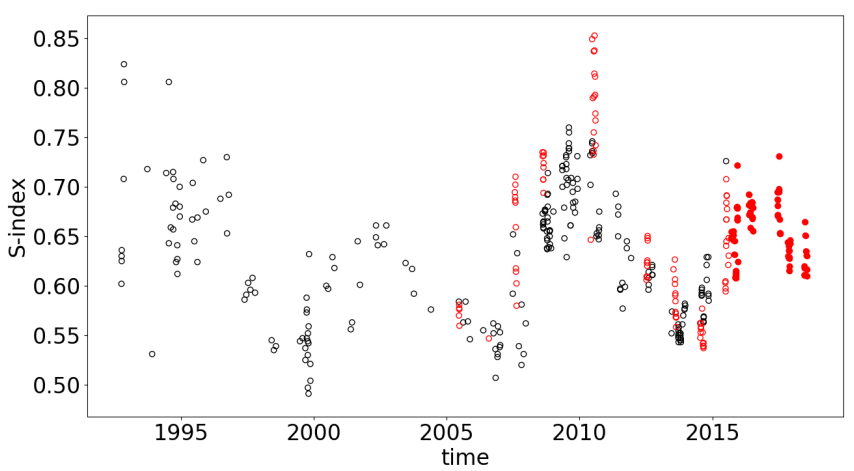

Fig. 1. S-index of 61 Cyg A measured using data from NARVAL/TBL and Lowell observatory (taken from Paper I) together with new measurements from this work. The Lowell data is shown as black open circles and the old NARVAL/TBL data is shown as red open circles. The red filled circles are data from this work.

$l_{\max }$ of 11 is applied to all of our maps ${ }^{3}$. The differential rotation parameter determined in Paper I is applied here. To determine differential rotation ZDI requires a dense phase coverage (Morgenthaler et al. 2012) and a complex field geometry so that the code can track different magnetic features as the star rotates and determine the surface shear. The field geometry of $61 \mathrm{Cyg}$ $\mathrm{A}$ is predominantly dipolar with single polarity in the observed hemisphere. Since there is no change in surface geometry in the visible hemisphere it is extremely hard to converge to the correct differential rotation. We have thus used the differential rotation from activity cycle I (Paper I). Using the differential rotation parameters from activity cycle I, a reduced $\chi^{2}$ of 1.0 is achieved for all epochs except 2016.50, where the reduced $\chi^{2}$ is 1.2 .

\section{Results}

\subsection{Chromospheric activity cycle II}

The chromospheric activity ( $\mathrm{S}$-index) is determined using the same method as in Paper I. Figure 1 shows that $61 \mathrm{Cyg}$ A has a periodic activity cycle of varying amplitude. The current cycle has a lower amplitude compared to cycle I. The length of cycle II also appears to be shorter than activity cycle I. However, cycle II is not over and new observations will provide a better estimate of its length.

\subsection{Magnetic geometry}

ZDI maps of $61 \mathrm{Cyg}$ A in cycle II (this work) are dominantly poloidal. The poloidal field is concentrated at lower order spherical harmonics: dipolar, quadrupolar, and octupolar, as shown in Fig. 2 (Fig. A.3 shows the poloidal magnetic energy distribution over the magnetic cycle of 61 Cyg A, epoch 2005.472018.52). More than $80 \%$ of the poloidal energy is dipolar except in 2016.50, close to activity maximum in cycle II (Table A.1).

From activity minimum to maximum. During the first two epochs $(2015.77,2015.91)$ the activity increases and moves towards activity maximum. During this period the radial field exhibits a strong positive polarity dipole in the observed hemisphere, as shown in Fig. A.1. The azimuthal field shows a strong band of negative polarity at equatorial latitudes in 2015.77, which

\footnotetext{
3 A $l_{\max }$ value of 11 was also applied in Paper I. As the majority of the magnetic energy is in the lower order components, $l_{\max } \geq 11$ does not improve the quality of the maps.
} 


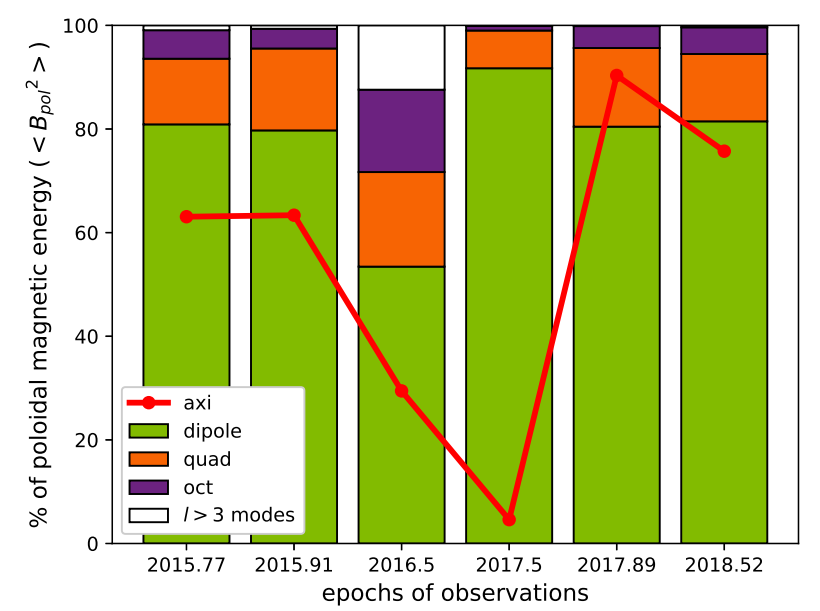

Fig. 2. Magnetic energy distribution in different components of the 61 Cyg A poloidal field during the current activity cycle (cycle II). Magnetic energy in the dipolar component is shown in green, the quadrupolar component is shown in orange, the octupolar component is shown in purple, and higher order components $(l>3)$ are shown in white. The red line shows the axisymmetry of the total field.

gets weaker in 2015.91. The meridional field shows a positive polarity band also at equatorial latitudes that remains consistent.

Near activity maximum. During epoch 2016.50 the largescale field appears to convert from a simple dipolar geometry (Fig. A.1). The azimuthal field gets stronger and the dipolar energy gets weaker at this epoch. A small fraction of the magnetic energy is detected in $l_{\max }>3$. The higher order modes were also detected at activity maximum during activity cycle I.

At epoch 2017.50, the radial field is reconstructed with both strong negative and positive regions (Fig. A.2), indicating a dipole whose axis is aligned to the equator. The meridional and azimuthal fields are considerably weaker. The field is almost purely poloidal and the dipole energy is at its strongest. At 2017.50 the negative polarity of the dipole gets stronger, suggesting a change in the dominating polarity in the coming epochs.

From activity maximum to minimum. During epochs 2017.89 and 2018.52 the radial field switches polarity; with the appearance of a strong negative dipolar field at the visible hemisphere (Fig. A.2). The meridional field also exhibits a negative polarity field, but at equatorial latitudes. The azimuthal field appears as a more complex field in 2018.52 (see Appendix A for a more detailed description on the field geometry).

\subsection{Dipole migration}

One key observation from Figs. A.1 and A.2 is the changing inclination of the radial dipolar field. In order to quantify the inclination of the dipole we calculated the location of the dipole axis for all available epochs, starting with 2005.47 up to 2018.52 . The axis of the dipole is obtained by finding the maximum of the dipole field (positive pole). Figure 3 shows the location of the dipolar axis, where it moves from one hemisphere to the other and comes back to the original hemisphere during a full magnetic cycle. This evolving nature of the dipolar axis location is a common feature of the solar large-scale field (DeRosa et al. 2012).

\subsection{Dipolar field and stellar activity}

Figure 4 shows the evolution of the dipole latitude and strength in conjunction with the $\mathrm{S}$-index cycle. The data for cycle $\mathrm{I}$ is

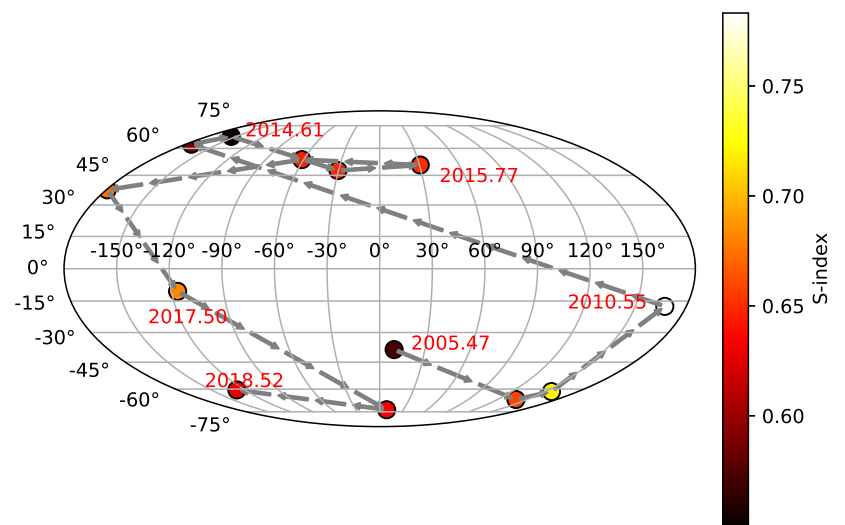

Fig. 3. Location of the dipole axis during activity cycle I and II ( 1 magnetic cycle), starting at the lower right and moving anti-clockwise. All values except 2005 are taken from this work or Paper I. The colour bar represents the chromospheric activity index $\mathrm{S}$-index. The arrow shows the direction of the dipole axis movement from 2005.47 to 2018.52 . Some representative dates are shown in red.

taken from Paper I. Mean S-indices are shown for each epoch. The standard deviations are shown as error bars. Cycle minima are shown as pink vertical bars and cycle maxima as green bars. As the exact occurrence of activity maximum is not known, it could be anytime in the one-year period around the second green vertical bar in Fig. 4.

In Fig. 4 we are missing two data points in the bottom two subplots, 2006 and 2012. Only one spectropolarimetric observation was taken in 2006 , first minimum, which is not enough to reconstruct a magnetic map. The spectropolarimetric observations in 2012 had polarisation anomalies (see Paper I for more details); as a result, the magnetic map could not be reconstructed. As shown in Fig. 4, the dipolar axis is located near polar latitudes at activity minimum and at equatorial latitudes at activity maximum. Although we do not have any data points at first minimum and second maximum (Fig. 4 middle), the overall trend in dipole location shows that there is a strong correlation with activity cycle phase, and both exhibit periodic behaviour. The dipole axis evolution agrees with a magnetic cycle period of $\sim 14$ years.

The strength of the dipole, $B_{\text {dipole }}$, is also plotted against time, as shown in the bottom subplot in Fig.4. The maximum of the dipole field, which occurs at the dipole axis, is taken to be the dipole strength in Fig. 4. We do not have a magnetic map for the first minimum in cycle I; 2007 and 2005 are the closest to the first minimum in activity cycle I. The dipolar field is at its weakest at the first maximum. During the second minimum the dipole increases in strength. Although the peak of the second maximum is not observed, close to the second activity maximum the dipole field remains relatively strong. No correlation is detected between the dipole field strength and the magnetic cycle.

Figure 5 shows the vector field strength of the axisymmetric dipole $(l=1, m=0)$ in both poloidal and toroidal components (similar to Fig. 11 in Paper I). The poloidal axisymmetric dipole is calculated by taking the maximum of the vector $B(l=1, m=0)$ at the visible rotational pole. The toroidal axisymmetric dipole is calculated by measuring the maximum signed $B(l=1, m=0)$ at the nearest toroidal band to the rotational pole. The poloidal dipole exhibits cyclic variation with a period that correlates to the dipole axis location in Fig. 4. The sign of the poloidal dipole field changes with the magnetic cycle phase. The toroidal dipole exhibits a weak anti-correlation with 

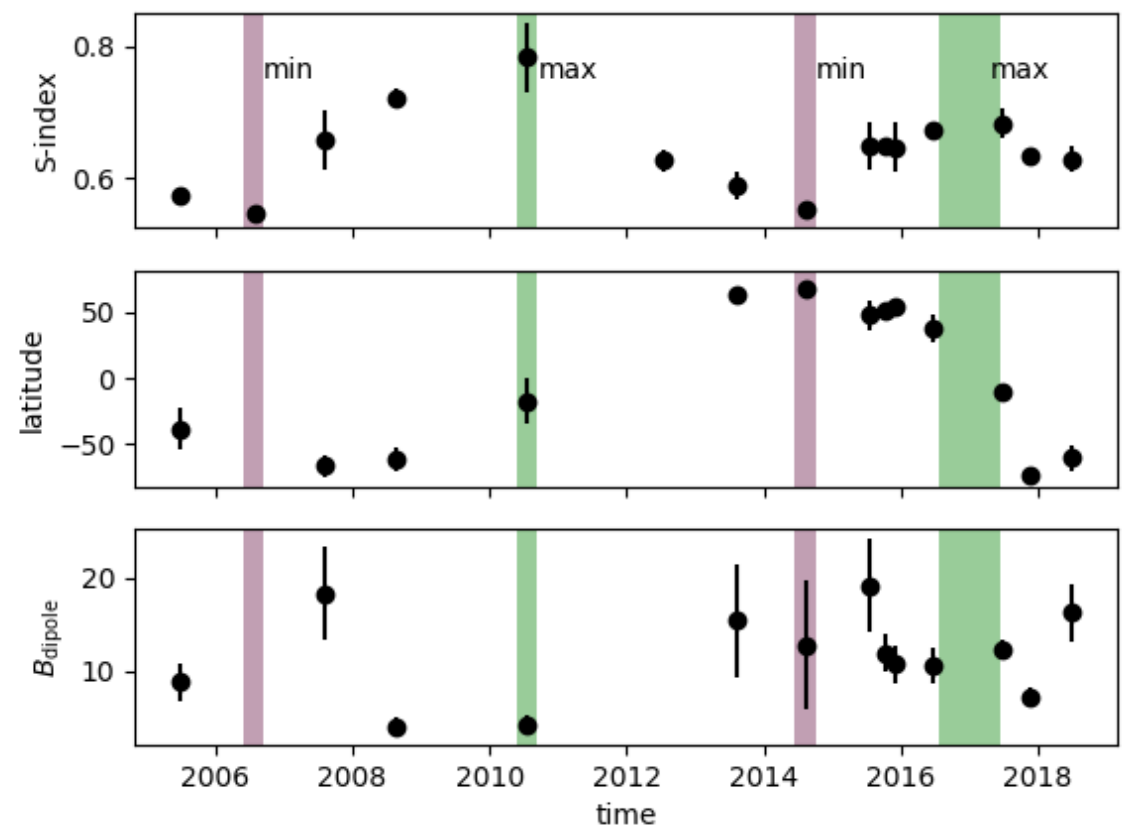

Fig. 4. Chromospheric activity (S-index, top panel) cycle, dipole location (middle panel), and strength (bottom panel) over time. Top panel: S-index cycle, middle panel: dipole location and bottom panel: dipole strength at the axis as a function of time. The standard deviation is shown as an error bar. Activity minimum is shown in purple and activity maximum is shown in green. the poloidal dipole field. The errors in Figs. 4 (middle and bottom panel) and 5 are determined via the same method used in Paper I (see Sect. 5.1).

\subsection{Evidence of the dipole flip in LSD Stokes $V$ profiles}

The dipole flip of $61 \mathrm{Cyg} \mathrm{A}$ is also seen directly in the LSD Stokes $V$ profiles. Figure B. 1 shows the LSD Stokes $V$ profiles from epochs 2015.77-2018.52 in the same scale. The Stokes $V$ profiles are shifted and colour-scaled to their rotational phase. Although the observed phases are not uniform in every single epoch, there are multiple observations with similar phases enabling direct comparison between the epochs. During epochs of single dominant polarity at the observed hemisphere (in the ZDI maps), the dominant Stokes $V$ profiles all share the same sign, with a positive lobe on one side of the line and a negative lobe on the other, although the amplitude may vary. During epochs of mixed polarity on the visible hemisphere, the Stokes $V$ profiles exhibit both positive and negative signs. The epochs 2015.77 and 2015.91 show dominant positive Stokes $V$ profiles, whereas epochs 2016.50 and 2017.50 show both positive and negative profiles. Finally epoch 2017.89 and 2018.52 show dominant negative Stokes $V$ profiles. This change in sign provides model independent evidence of the large-scale field polarity reversal (see Appendix B).

\section{Discussion and conclusions}

The observations taken in this work in combination with archival data (Paper I + Petit et al. 2014) result in an observational time span of $\sim 14$ years, providing a unique opportunity to monitor the large-scale surface magnetic geometry of 61 Cyg A over its magnetic cycle (two simultaneous activity cycles). The current activity cycle has a smaller amplitude and length than activity cycle I (Paper I). This shows that although the cycle is quasiperiodic, it is not uniform in length. Previous observations of the star's cycle have also shown this non-uniformity in amplitude and period length (Baliunas et al. 1995; also see Paper I), although the period length does not vary dramatically for a short amplitude cycle. Further observations of the current cycle are

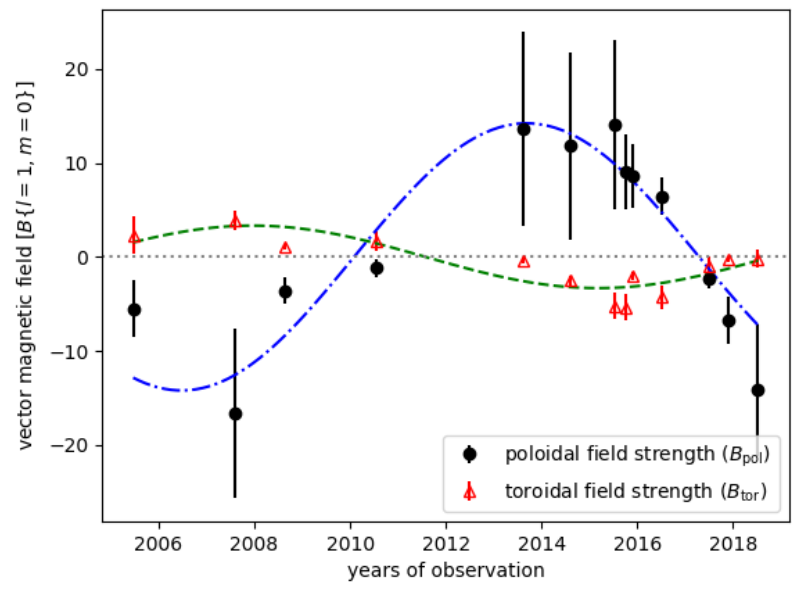

Fig. 5. Axisymmetric dipole field strength, $B(l=1, m=0)$, both poloidal (black filled circles) and toroidal (red open triangles) components of the magnetic field. The dotted horizontal line in grey represents $B=0$. The sinusoidal fits are obtained with a magnetic cycle period of 14.4 years. The error bars are dispersion obtained in a way similar to that in Paper I. This is a continuation of Fig. 11 in Paper I.

required to determine the true cycle period of cycle II. The solar cycle is also known to vary in length (Hathaway 2010).

The large-scale field of the star is strongly poloidal throughout cycle II. The poloidal field is constituted of mostly lower order spherical harmonics, of which the dipole is the strongest. The dipole remains consistently dominant throughout the observations except close to activity maximum. We do not have observations at the peak of maximum during cycle II, yet the dipole field gets considerably weaker during epoch 2016.50. At epoch 2016.50 the quadrupolar, octupolar, and higher order components have higher energies compared to other epochs. Similar weakening of the dipole was also detected during activity maximum in cycle I.

Similar to the solar case, the large-scale dipole flips its polarity in sync with the chromospheric activity cycle in a quasiperiodic manner. The dipole field reverts to its original polarity during our combined $\sim 14$ years of observation. The dipole axis 
location has a tight correlation with the star's magnetic cycle. The axisymmetric vector dipole field also evolves together with magnetic cycle indicating a period of $\sim 14$ years. Overall our results show that the evolution of the $61 \mathrm{Cyg}$ A large-scale field, specifically the dipole field, is very similar to the solar case indicating common dynamo processes. The solar magnetic cycle, in the large-scale, could be a template for sun-like stars like $61 \mathrm{Cyg}$ $A$ and vice versa.

Acknowledgements. We thank Matthew Mengel for providing us with some of the data and the TBL team for their help during the observations. S.B.S and T.L. acknowledge funding via the Austrian Space Application Programme (ASAP) of the Austrian Research Promotion Agency (FFG) within ASAP11, the FWF NFN project S11601-N16, and the sub-project S11604-N16. S.V.J acknowledges the support of the German Science Foundation (DFG) Research Unit FOR2544 "Blue Planets around Red Stars", project JE 701/3-1, and DFG priority program SPP 1992 "Exploring the Diversity of Extrasolar Planets (RE 1664/18)". V.S. acknowledges funding from the European Research Council (ERC) under the European Union's Horizon 2020 research and innovation programme (grant agreement No. 682393 AWESoMeStars). A.A.V. acknowledges funding received from the Irish Research Council Laureate Awards 2017/2018.

\section{References}

Aurière, M. 2003, in EAS Pub. Ser., eds. J. Arnaud, \& N. Meunier, 9, 105 Baliunas, S. L., Donahue, R. A., Soon, W. H., et al. 1995, ApJ, 438, 269

Boro Saikia, S., Jeffers, S. V., Petit, P., et al. 2015, A\&A, 573, A17

Boro Saikia, S., Jeffers, S. V., Morin, J., et al. 2016, A\&A, 594, A29

Brown, S. F., Donati, J.-F., Rees, D. E., \& Semel, M. 1991, A\&A, 250, 463
DeRosa, M. L., Brun, A. S., \& Hoeksema, J. T. 2012, ApJ, 757, 96

Donati, J.-F., \& Brown, S. F. 1997, A\&A, 326, 1135

Donati, J.-F., Semel, M., Carter, B. D., Rees, D. E., \& Collier Cameron, A. 1997, MNRAS, 291, 658

Donati, J.-F., Howarth, I. D., Jardine, M. M., et al. 2006, MNRAS, 370, 629

Fares, R., Donati, J.-F., Moutou, C., et al. 2009, MNRAS, 398, 1383

Fares, R., Bourrier, V., Vidotto, A. A., et al. 2017, MNRAS, 471, 1246

Finley, A. J., Matt, S. P., \& See, V. 2018, ApJ, 864, 125

Folsom, C. P., Petit, P., Bouvier, J., et al. 2016, MNRAS, 457, 580

Hackman, T., Lehtinen, J., Rosén, L., Kochukhov, O., \& Käpylä, M. J. 2016, A\&A, 587, A28

Hathaway, D. H. 2010, Liv. Rev. Sol. Phys., 7, 1

Jeffers, S. V., Petit, P., Marsden, S. C., et al. 2014, A\&A, 569, A79

Jeffers, S. V., Boro Saikia, S., Barnes, J. R., et al. 2017, MNRAS, 471, L96

Jeffers, S. V., Mengel, M., Moutou, C., et al. 2018, MNRAS, 479, 5266

Kochukhov, O., \& Piskunov, N. 2002, A\&A, 388, 868

Kochukhov, O., Makaganiuk, V., \& Piskunov, N. 2010, A\&A, 524, A5

Marsden, S. C., Petit, P., Jeffers, S. V., et al. 2014, MNRAS, 444, 3517

Mengel, M. W., Fares, R., Marsden, S. C., et al. 2016, MNRAS, 459, 4325

Morgenthaler, A., Petit, P., Morin, J., et al. 2011, Astron. Nachr., 332, 866

Morgenthaler, A., Petit, P., Saar, S., et al. 2012, A\&A, 540, A138

Petit, P., Dintrans, B., Solanki, S. K., et al. 2008, MNRAS, 388, 80

Petit, P., Dintrans, B., Morgenthaler, A., et al. 2009, A\&A, 508, L9

Petit, P., Louge, T., Théado, S., et al. 2014, PASP, 126, 469

Piskunov, N., \& Kochukhov, O. 2002, A\&A, 381, 736

Rosén, L., Kochukhov, O., Hackman, T., \& Lehtinen, J. 2016, A\&A, 593, A35

Sanderson, T. R., Appourchaux, T., Hoeksema, J. T., \& Harvey, K. L. 2003, J. Geophys. Res. (Space Physics), 108, 1035

Semel, M. 1989, A\&A, 225, 456

Skilling, J., \& Bryan, R. K. 1984, MNRAS, 211, 111

Vidotto, A. A. 2016, MNRAS, 459, 1533

Vidotto, A. A., Lehmann, L. T., Jardine, M., \& Pevtsov, A. A. 2018, MNRAS, 480, 477 


\section{Appendix A: Surface magnetic maps}

Table A.1. Magnetic energy in the different magnetic field components.

\begin{tabular}{cccccccc}
\hline \hline epoch & $\begin{array}{c}\text { Bmean } \\
(\mathrm{G})\end{array}$ & $\begin{array}{c}\text { pol } \\
(\% \text { tot })\end{array}$ & $\begin{array}{c}\text { dipole } \\
(\% \text { pol })\end{array}$ & $\begin{array}{c}\text { quad } \\
(\% \text { pol })\end{array}$ & $\begin{array}{c}\text { oct } \\
(\% \text { pol })\end{array}$ & $\begin{array}{c}\text { axi } \\
(\% \text { tot })\end{array}$ & S-index \\
\hline 2015.77 & $9 \pm 1$ & $74 \pm 12$ & $81 \pm 10$ & $13 \pm 5$ & $5 \pm 3$ & $63 \pm 7$ & $0.647 \pm 0.007$ \\
2015.91 & $6 \pm 1$ & $95 \pm 1$ & $80 \pm 6$ & $16 \pm 6$ & $3 \pm 1$ & $63 \pm 9$ & $0.65 \pm 0.04$ \\
2016.50 & $9 \pm 2$ & $79 \pm 5$ & $53 \pm 11$ & $18 \pm 4$ & $15 \pm 2$ & $29 \pm 6$ & $0.67 \pm 0.01$ \\
2017.50 & $7 \pm 1$ & $99_{-3}^{+1}$ & $92_{-9}^{+8}$ & $7 \pm 8$ & $1_{-1}^{+2}$ & $5 \pm 2$ & $0.68 \pm 0.02$ \\
2017.89 & $4 \pm 1$ & $99 \pm 1$ & $80 \pm 7$ & $15 \pm 5$ & $4 \pm 2$ & $90 \pm 1$ & $0.635 \pm 0.009$ \\
2018.52 & $10 \pm 2$ & $99 \pm 1$ & $81 \pm 12$ & $13 \pm 4$ & $5_{-5}^{+6}$ & $75 \pm 22$ & $0.63 \pm 0.02$ \\
\hline
\end{tabular}

Notes. The fractional dates are shown in Col. 1, followed by the mean magnetic field (in gauss) in Col. 2. Column 3 is the poloidal energy, Col. 4 is the poloidal dipolar energy, Col. 5 is the poloidal quadrupolar energy, Col. 6 is the poloidal octupolar energy, Col. 7 is the axisymmetry of the total field. The error bars are obtained using the same method as in Paper I (Sect. 5.1). Finally, Col. 8 is the mean S-index and the standard deviation as error bars.

Figures A.1 and A.2 show the radial, meridional, and azimuthal field of the star during activity cycle II. The magnetic maps are shown in flattened polar projection.

The first two epochs $(2015.77,2015.91)$ were observed within a short period of four months (see Table C.1) during intermediate chromospheric activity. The radial field (Fig. A.1, top) exhibits a strong positive polarity magnetic region between the equator and higher latitudes, indicating a dipolar geometry. The overall structure of the field does not change during the two epochs. The meridional field also remains consistent during the two epochs with a strong band of positive polarity field at equatorial latitudes. Contrary to the radial and meridional field, the azimuthal field has a band of negative polarity field that changes in size within such a short period of time.

Table A.1 shows that the poloidal energy changes by $~ 20 \%$. This change in the poloidal energy could be attributed to the strong azimuthal field in 2015.77. Despite a weak poloidal component in 2015.77 , the dipole energy is strong. The axisymmetry of the field is also relatively strong during these two epochs (Table A.1). The increase in the strength of the azimuthal field is not reflected in the complexity of the field.

Epochs 2016.50 and 2017.50 were taken close to activity maximum in cycle II. The S-index cycle in Fig. 1 shows that our observations were taken before and after the peak of activity maximum. As the previous cycle (cycle I) had a period length of 7.2 years, activity maximum was not expected during these two epochs. Nonetheless, it is quite clear that activity maximum occurred between 2016.50 and 2017.50. Both of these epochs have similar levels of activity, but the field geometry of 2016.50 epoch is more representative of activity maximum than epoch 2017.50. During 2016.50 the large-scale field appears more complex, as shown in Fig. A.1. This increase in complexity is also seen in the weakening dipole energy, followed by the increase in strength of higher order spherical harmonics. The strong positive polarity magnetic region from previous epochs gets smaller and a negative polarity region appears at equatorial latitudes. The meridional field also appears to have a more complex geometry with both positive and negative field regions at the poles and near the equator. The azimuthal field has a dominant negative polarity with a small positive polarity region.

The large-scale field geometry changes drastically in epoch 2017.50. The complex field geometry from 2016.50 is taken over by a simple dipolar geometry where the dipolar energy is at $92 \%$. The radial dipole is aligned to the equator, which is shown by the appearance of both polarities in the visible hemisphere. Weak positive and negative polarities are also detected in the meridional and azimuthal field.

At maximum activity, the solar surface is dominated by small bipolar magnetic regions. When the solar field geometry is decomposed into its lower spherical harmonic modes, at maximum, it also appears to have strong opposite polarity magnetic regions at the equator (Vidotto 2016; Vidotto et al. 2018). The radial field geometry during 2016.50 and 2017.50 shows strong similarities with the solar large-scale field at activity maximum.

The appearance of both positive and negative fields around equatorial latitudes indicates that the star's activity is changing drastically. The visible hemisphere will soon switch polarity from positive to negative. Unsurprisingly, we observe a strong negative polarity dipole in epoch 2017.89. This transition of the large-scale dipole field is also seen in the Sun (DeRosa et al. 2012; Finley et al. 2018). Recent analyses by Vidotto et al. (2018) indicate that the solar large-scale field, filtered for the large-scale components, shows a similar trend to that presented in Figs. A.1 and A.2.

During epochs 2017.89 and 2018.52 the activity decreases even further, as shown in Fig. 1. The change in polarity appears before the actual activity minimum. This is not surprising as in Paper I the polarity switch also occurred before the peak of activity minimum. Furthermore, the negative polarity magnetic field around the equator in the meridional component gets stronger with time. The azimuthal component increases in complexity during 2018.52. This might indicate that it could change polarity during the following epochs.

Our combined observations have shown that the star's largescale (radial and meridional) field changes polarity in phase with the chromospheric activity cycle. The close agreement between the radial and meridional field could also be attributed to crosstalk (Donati \& Brown 1997; Jeffers et al. 2017). It takes almost twice the length of the activity cycle (one magnetic cycle) for the radial and meridional field to switch back to its original polarity. Based on our observations, the azimuthal field switches polarity once during the magnetic cycle. Additionally, the large-scale field is strongly poloidal and the poloidal energy is concentrated mostly in lower order harmonics, as shown in Fig. A.3. The $l_{\max }>3$ modes dominate at or near the two activity maxima. The poloidal field also loses its axisymmetry close to activity maxima. The large-scale field evolution strongly indicates that the magnetic cycle is twice the length of the activity cycle. 
S. Boro Saikia et al.: Dipole
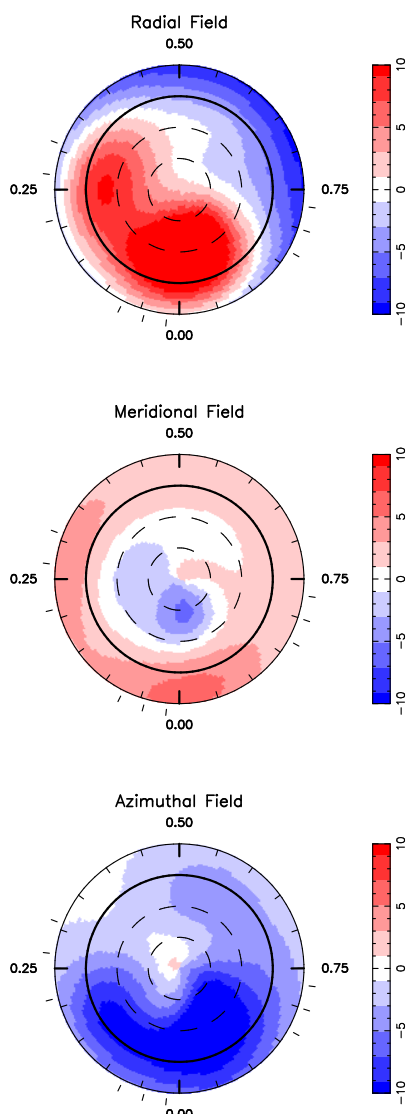

(a) 2015.77
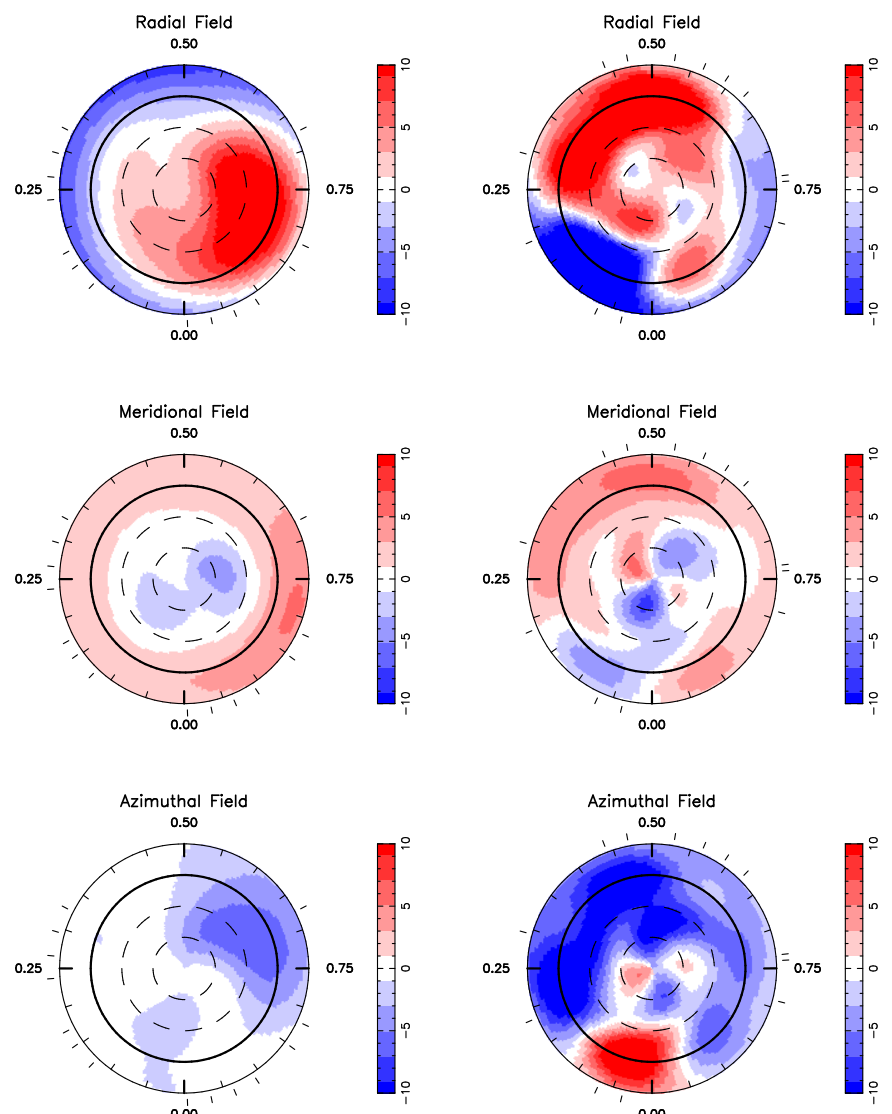

(b) 2015.91

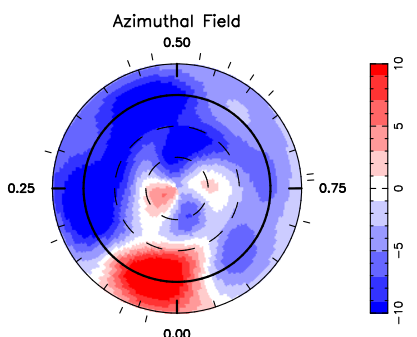

(c) 2016.50

Fig. A.1. ZDI maps of 61 Cyg A for epochs 2015.77, 2015.91, and 2016.50 (from left to right panels). The radial field is shown at the top, the meridional field in the middle, and the azimuthal field at the bottom. Flatted polar projections are shown that go down to latitudes of $-30^{\circ}$. The solid black line is the equator. The small black tick marks represent the observed rotational phases. The positive and negative fields (in gauss) are shown in red and blue, respectively. 

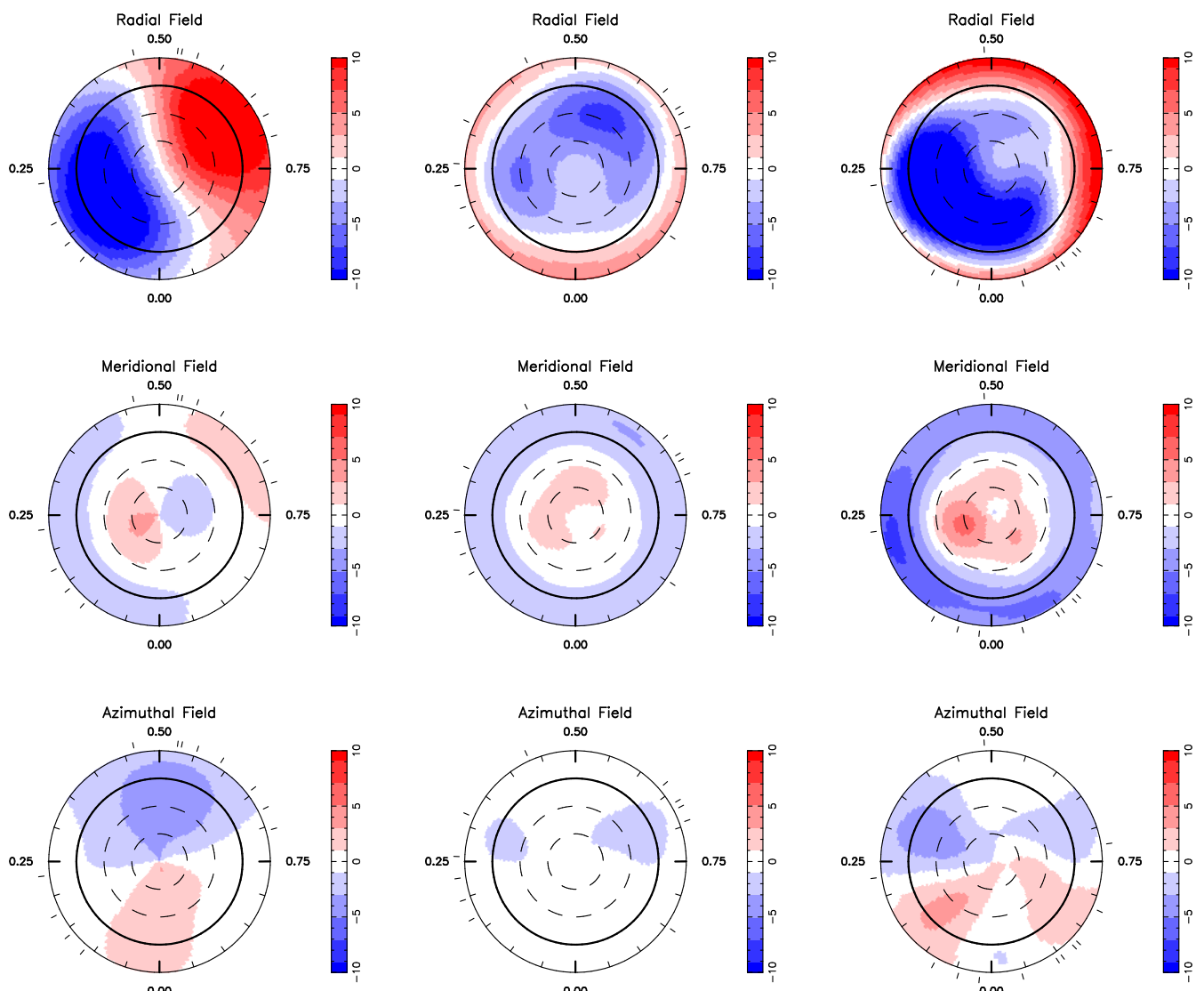

(a) 2017.50

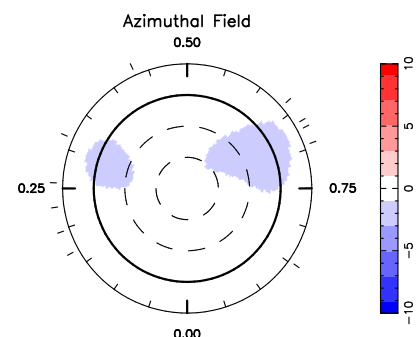

(b) 2017.89

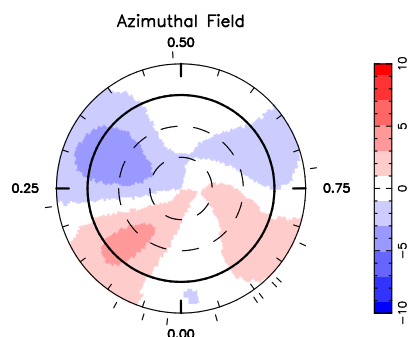

(c) 2018.52

Fig. A.2. ZDI maps of 61 Cyg A for epochs 2017.50, 2017.89, and 2018.52. Colours and symbol as in Fig. A.1.

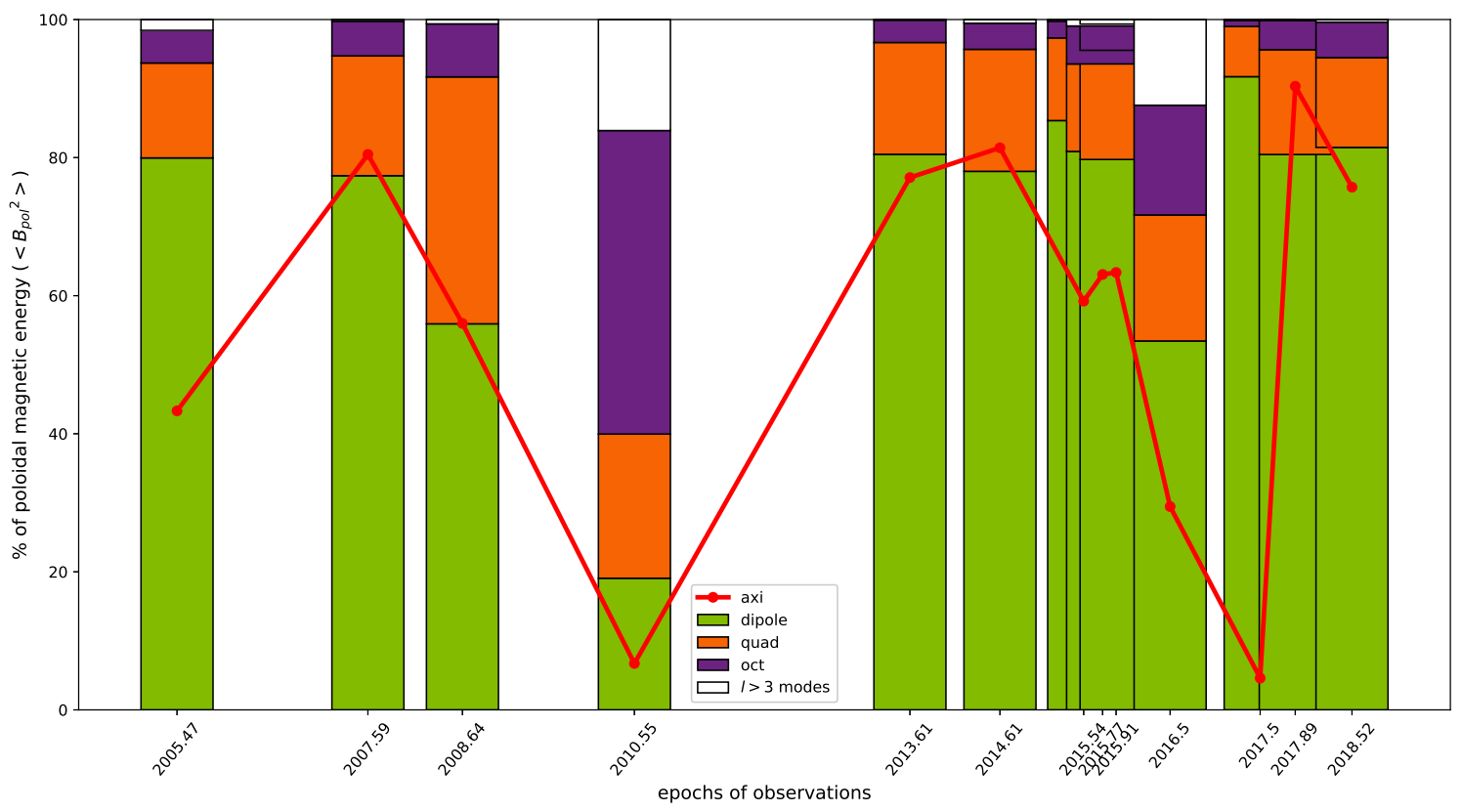

Fig. A.3. Same as Fig. 2, but for the entire magnetic cycle. The $x$-axis is scaled to time. Epochs 2007.59-2015.54 were previously published in Paper I. 
Appendix B: Dipole flip as seen in Stokes $V$ profiles

The LSD Stokes $V$ spectra and fits associated with the ZDI maps from Figs. A.1 and A.2 are shown in Fig. B.1. It provides a ZDI independent look at the field complexity evolution during activity cycle II. The amplitude of the profile indicates the magnetic field strength and the field polarity is determined by the sign of the profile. As shown in Fig. B.1, during the first two epochs in cycle II the stronger profiles all have the same sign. Thus, it is quite clear that a single polarity dominates the visible hemisphere during these epochs. Although all Stokes $V$ profiles per epoch were not observed at the same rotational phases, the dominant Stokes $V$ profiles observed at similar phases all have the same sign, suggesting a dominant polarity at the visible hemisphere. As shown by the ZDI maps in Fig. A.1, the dominant polarity is positive.

Close to cycle maximum (epochs 2016.50, 2017.50) the Stokes $V$ signals exhibit mixed signs, which indicate that both positive and negative polarities are present and which one dominates (i.e. is the most visible) depends on rotation phase. The amplitudes of these oppositely aligned Stokes $V$ signals are comparably strong. The profiles with the same sign are located close to each other in phase. In 2016.50, however, the profiles with a positive sign (facing right) have a better phase coverage and dominate over the negative profiles. This is represented in the reconstructed map as a stronger positive polarity and a small negative polarity in the radial field in epoch 2016.50. During 2017.50 both positive and negative profiles have similar amplitude and phase coverage. This indicates that one half of the stellar surface is dominated by one polarity and the other half of the surface is dominated by another polarity. This is also seen in the ZDI map (Fig. A.2).

Finally, during the last two epochs $(2017.89,2018.52)$ the Stokes $V$ line profiles all have the same sign, indicating a single polarity field. Additionally, the profiles are facing the opposite sign as the first two epochs. Thus, clearly the dominant magnetic field in the visible portions of the star has reversed sign. These two epochs have profiles that were taken during similar rotational phases and all of these profiles face the same sign. Some of the profiles in 2015.91 and 2018.52 have similar rotational phases, and as expected their signs are opposite. This strongly agrees with the ZDI maps in Fig. A.2. The LSD Stokes $V$ profiles show clear agreement with the ZDI reconstructed maps providing additional confidence to the $\mathrm{ZDI}$ technique. 
A\&A 620, L11 (2018)

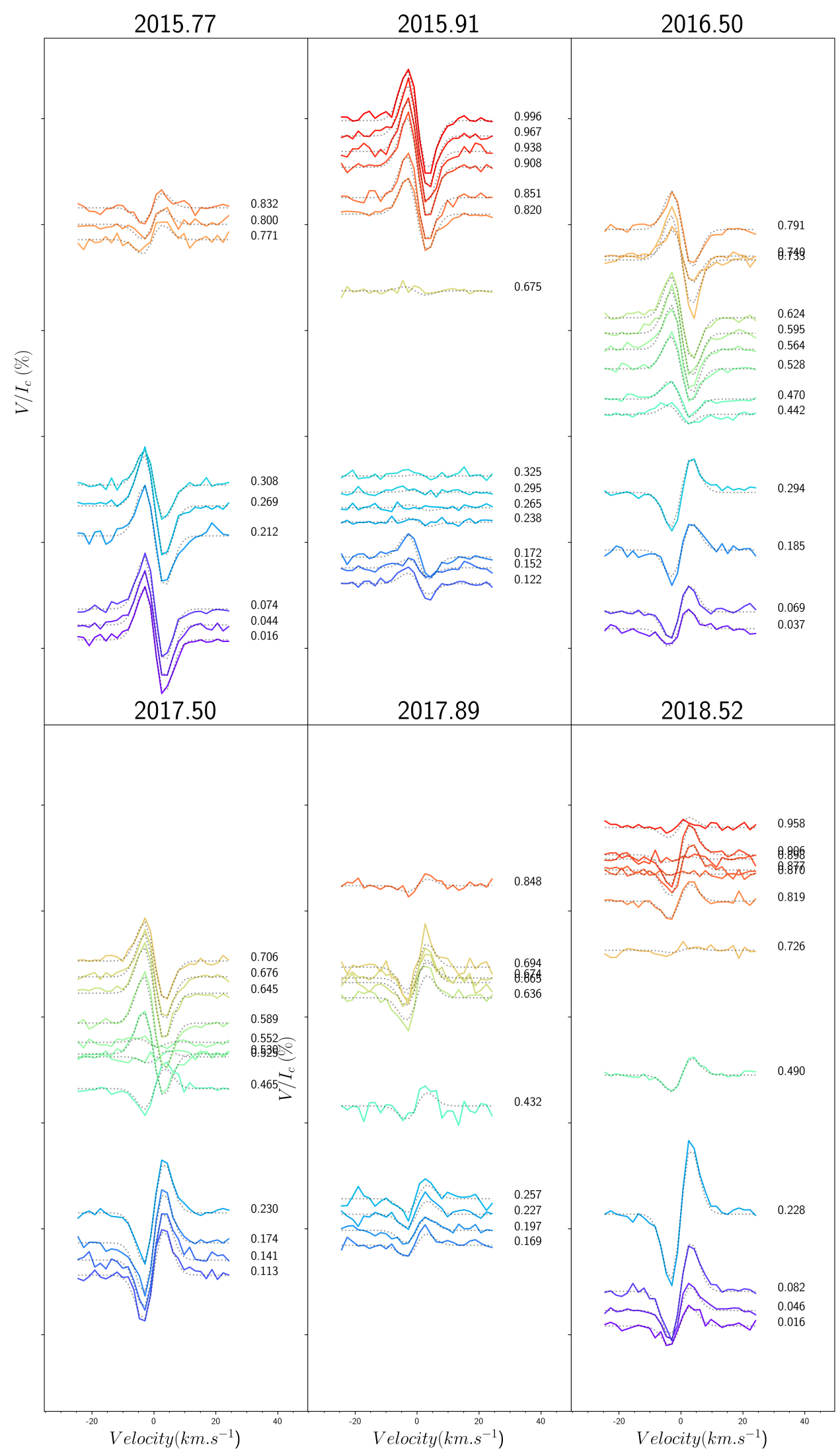

Fig. B.1. Stokes V LSD spectra of 61 Cyg A. Top panel: epochs 2015.77, 2015.91, and 2016.50. Bottom panel: epochs 2017.50, 2017.89, and 2018.52 (from left to right). The observed profile is shown in colour and the fit is shown as dotted lines. The Stokes $V$ profiles are shifted according to their phase, where the phase increases from 0 to 1 . The colour bar also changes with phase. The colour bar and scale of each subplot is kept the same. 


\section{Appendix C: Journal of observations}

Table C.1. Journal of observations for the six epochs in this work.

\begin{tabular}{|c|c|c|c|c|c|c|}
\hline Epoch & Date & $\begin{array}{c}\text { Julian date } \\
(2450000+)\end{array}$ & $\begin{array}{c}\text { Exp. time } \\
\text { (s) }\end{array}$ & LSD $S / N$ & $\begin{array}{c}\sigma_{\mathrm{LSD}} \\
10^{-5} I_{\mathrm{c}} \\
\end{array}$ & $\begin{array}{c}\text { Rotational } \\
\text { phase }\end{array}$ \\
\hline \multirow{9}{*}{2015.77} & 18 September 2015 & 7284.43644 & 800 & 32619 & 3.07 & 0.016 \\
\hline & 19 September 2015 & 7285.39578 & 800 & 29201 & 3.42 & 0.044 \\
\hline & 20 September 2015 & 7286.42822 & 800 & 30034 & 3.33 & 0.074 \\
\hline & 28 September 2015 & 7294.43000 & 800 & 30351 & 3.29 & 0.308 \\
\hline & 14 October 2015 & 7310.27758 & 800 & 21896 & 4.57 & 0.771 \\
\hline & 15 October 2015 & 7311.26826 & 800 & 30609 & 3.27 & 0.800 \\
\hline & 16 October 2015 & 7312.33382 & 800 & 25388 & 3.94 & 0.832 \\
\hline & 29 October 2015 & 7325.34651 & 800 & 24249 & 4.12 & 0.212 \\
\hline & 31 October 2015 & 7327.28473 & 800 & 40144 & 2.49 & 0.269 \\
\hline \multirow{14}{*}{2015.91} & 12 November 2015 & 7339.36547 & 800 & 33834 & 2.96 & 0.122 \\
\hline & 13 November 2015 & 7340.39006 & 800 & 30554 & 3.27 & 0.152 \\
\hline & 16 November 2015 & 7343.33408 & 800 & 31471 & 3.18 & 0.238 \\
\hline & 17 November 2015 & 7344.26886 & 800 & 36146 & 2.77 & 0.265 \\
\hline & 18 November 2015 & 7345.27477 & 800 & 36311 & 2.75 & 0.295 \\
\hline & 19 November 2015 & 7346.32211 & 800 & 31212 & 3.20 & 0.325 \\
\hline & 01 December 2015 & 7358.27061 & 800 & 33825 & 2.96 & 0.675 \\
\hline & 06 December 2015 & 7363.22625 & 800 & 35305 & 2.83 & 0.820 \\
\hline & 07 December 2015 & 7364.29177 & 800 & 25713 & 3.88 & 0.851 \\
\hline & 09 December 2015 & 7366.25934 & 800 & 30635 & 3.26 & 0.908 \\
\hline & 10 December 2015 & 7367.27495 & 800 & 23688 & 4.22 & 0.938 \\
\hline & 11 December 2015 & 7368.27652 & 800 & 35735 & 2.80 & 0.967 \\
\hline & 12 December 2015 & 7369.26755 & 800 & 30829 & 3.24 & 0.996 \\
\hline & 18 December 2015 & 7375.27907 & 800 & 35732 & 2.80 & 0.172 \\
\hline \multirow{13}{*}{2016.50} & 15 May 2016 & 7524.55989 & 900 & 34639 & 2.89 & 0.037 \\
\hline & 16 May 2016 & 7525.63982 & 900 & 40526 & 2.47 & 0.069 \\
\hline & 20 May 2016 & 7529.63823 & 900 & 31859 & 3.14 & 0.185 \\
\hline & 02 June 2016 & 7542.59157 & 900 & 34959 & 2.86 & 0.564 \\
\hline & 03 June 2016 & 7543.63071 & 900 & 33443 & 2.99 & 0.595 \\
\hline & 04 June 2016 & 7544.63360 & 900 & 38889 & 2.57 & 0.624 \\
\hline & 08 June 2016 & 7548.61223 & 900 & 44345 & 2.26 & 0.740 \\
\hline & 27 June 2016 & 7567.55244 & 900 & 39411 & 2.54 & 0.294 \\
\hline & 02 July 2016 & 7572.59730 & 900 & 38673 & 2.59 & 0.442 \\
\hline & 03 July 2016 & 7573.58564 & 900 & 45863 & 2.18 & 0.470 \\
\hline & 05 July 2016 & 7575.54213 & 900 & 40408 & 2.47 & 0.528 \\
\hline & 12 July 2016 & 7582.56750 & 900 & 22195 & 4.51 & 0.733 \\
\hline & 14 July 2016 & 7584.53737 & 900 & 39681 & 2.52 & 0.791 \\
\hline \multirow{12}{*}{2017.50} & 12 June 2017 & 7917.63192 & 800 & 33537 & 2.98 & 0.530 \\
\hline & 14 June 2017 & 7919.62389 & 800 & 32305 & 3.09 & 0.589 \\
\hline & 16 June 2017 & 7921.55409 & 800 & 34387 & 2.91 & 0.645 \\
\hline & 17 June 2017 & 7922.60711 & 800 & 34018 & 2.94 & 0.676 \\
\hline & 18 June 2017 & 7923.63342 & 800 & 34056 & 2.94 & 0.706 \\
\hline & 02 July 2017 & 7937.54560 & 800 & 22982 & 4.35 & 0.113 \\
\hline & 03 July 2017 & 7938.51815 & 800 & 26417 & 3.79 & 0.141 \\
\hline & 04 July 2017 & 7939.63532 & 800 & 24883 & 4.02 & 0.174 \\
\hline & 06 July 2017 & 7941.56775 & 800 & 31271 & 3.20 & 0.230 \\
\hline & 14 July 2017 & 7949.59151 & 800 & 26993 & 3.74 & 0.465 \\
\hline & 16 July 2017 & 7951.63701 & 800 & 21059 & 4.75 & 0.525 \\
\hline & 17 July 2017 & 7952.57869 & 800 & 26928 & 3.71 & 0.552 \\
\hline
\end{tabular}

Notes. From left to right: epoch, Heliocentric Julian date, exposure time in seconds, signal-to-noise ratio of the Stokes $V$ LSD profile, the error bar in Stokes $V$ LSD profile and the rotational phase. 
Table C.1. continued.

\begin{tabular}{ccccccc}
\hline \hline \multirow{2}{*}{ Epoch } & Date & $\begin{array}{c}\text { Julian date } \\
(2450000+)\end{array}$ & $\begin{array}{c}\text { Exp. time } \\
(\mathrm{s})\end{array}$ & LSD $S / N$ & $\begin{array}{c}\sigma_{\text {LSD }} \\
10^{-5} I_{\mathrm{c}}\end{array}$ & $\begin{array}{c}\text { Rotational } \\
\text { phase }\end{array}$ \\
\hline \multirow{5}{*}{2017.89} & 01 November 2017 & 8059.33560 & 900 & 18917 & 5.29 & 0.674 \\
& 07 November 2017 & 8065.30189 & 900 & 31161 & 3.21 & 0.848 \\
& 18 November 2017 & 8076.27407 & 900 & 26685 & 3.75 & 0.169 \\
& 19 November 2017 & 8077.23845 & 900 & 30423 & 3.29 & 0.197 \\
& 20 November 2017 & 8078.27494 & 900 & 23949 & 4.18 & 0.227 \\
& 21 November 2017 & 8079.27933 & 900 & 21370 & 4.68 & 0.257 \\
& 27 November 2017 & 8085.27097 & 900 & 14496 & 6.89 & 0.432 \\
& 04 December 2017 & 8092.25630 & 900 & 20306 & 4.93 & 0.636 \\
& 05 December 2017 & 8093.23727 & 900 & 18924 & 5.28 & 0.665 \\
& 06 December 2017 & 8094.23619 & 900 & 16058 & 6.23 & 0.694 \\
\hline \multirow{5}{*}{ 16 June 2018 } & 8286.59636 & 1200 & 33472 & 2.99 & 0.819 \\
& 18 June 2018 & 8288.60607 & 1200 & 29781 & 3.36 & 0.877 \\
& 19 June 2018 & 8289.59103 & 1200 & 39606 & 2.52 & 0.906 \\
& 25 June 2018 & 8295.59546 & 1200 & 31257 & 3.20 & 0.082 \\
& 30 June 2018 & 8300.58423 & 1200 & 35162 & 2.84 & 0.228 \\
& 09 July 2018 & 8309.56470 & 1200 & 40728 & 2.45 & 0.490 \\
& 17 July 2018 & 8317.62340 & 1200 & 34940 & 2.86 & 0.726 \\
& 22 July 2018 & 8322.54015 & 1200 & 32160 & 3.11 & 0.870 \\
& 23 July 2018 & 8323.51315 & 1200 & 24262 & 4.12 & 0.898 \\
& 25 July 2018 & 8325.55100 & 1200 & 28374 & 3.52 & 0.958 \\
& 27 July 2018 & 8327.56221 & 1200 & 28379 & 3.52 & 0.016 \\
28 July 2018 & 8328.56278 & 1200 & 34145 & 2.93 & 0.046 \\
\hline
\end{tabular}

